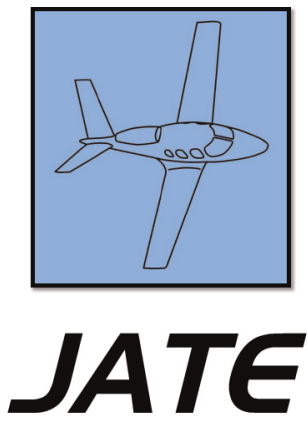

Journal of Aviation Technology and Engineering 2:1 (2012) 100-104

DOI: $10.5703 / 1288284314861$

\title{
Simulation Design Approach for the Selection of Alternative Commercial Passenger Aircraft Seating Configurations
}

\author{
Christopher A. Chung
}

University of Houston

\begin{abstract}
Loading strategies for commercial passenger aircraft have been a subject of recent study among air transportation research analysts. A fundamental assumption in the majority of these studies is the fixed configuration of passenger seats. Previous studies have focused on analyzing different strategies in an effort to reduce passenger loading time. This study takes a more proactive approach to the passenger loading process by starting with the design of the aircraft seating layout itself. Simulation analysis results indicate that alternative designs can result in loading time reductions between approximately $9-44 \%$.
\end{abstract}

Keywords: $\quad$ aircraft, seating

\section{Introduction}

The operational efficiency of commercial passenger aircraft can be characterized in many different manners. These include aerodynamic efficiency, cruising efficiency, fuel efficiency, propulsive efficiency, and structural efficiency (McLean, 2006). Absent from this list is passenger loading efficiency, which can be simply defined as the ratio of the number of loaded passengers divided by the aircraft loading time. This yields the number of passengers that can be loaded per unit time, such as minutes. Despite the fact that the loading process is a fundamental component of passenger aircraft operations, research has been limited toward passenger loading from a design perspective. What research has been performed has mainly focused on different strategies and algorithms involving predetermined seating configurations.

\footnotetext{
About the Authors

Christopher Chung is an associate professor in the Department of Industrial Engineering at the University of Houston. Dr. Chung performs research in the areas of simulation, simulators, and engineering management. He has received research funding from the Department of Homeland Security, the United States Coast Guard, the Department of Justice, and Continental Airlines. Dr. Chung received his B.E.S. in Materials Science and Engineering from Johns Hopkins University and his M.S.I.E. and Ph.D. in Industrial Engineering from the University of Pittsburgh.
} 
The objective of this research effort is to examine the operational effects of alternative passenger seating design configurations rather than passenger loading algorithms. This approach may provide exploratory insight in innovative ideas for designing new commercial passenger aircraft and selecting seating configurations for existing aircraft designs.

\section{Previous Relevant Research}

As previously discussed, the focus of commercial passenger aircraft loading research has been on strategies involving the passenger loading process of existing layouts. These efforts primarily include simulation modeling and optimization based analysis.

One early passenger loading research simulation based effort was conducted by Marelli, Mattocks, and Merry (1998). Though based on laboratory based input data, their Passenger Enplane/Deplane Simulation (PEDS) provided some insight into the loading process. Their tests verified that loading a commercial plane starting first with passenger assigned window seats and ending with aisle seats could significantly reduce aircraft loading times.

In another major research effort, Van Landeghem and Beuselinck (2002) examined the loading process of shorthaul flights with seating capacities between 80 and 150 passengers. Their analysis was limited to a "standard" layout with three seats on either side of a single airplane aisle. Their research results indicated the most effective loading would be achieved by individual passenger seating announcement. However, if that somewhat impractical approach proved too difficult to implement, the next most promising algorithm was by particular row and either port or starboard side. This meant that the left and right sides of the plane were loaded separately. This approach might also present loading problems for parties who were spread out across a particular row, as the party could not be seated at the same time.

Ferrari and Nagel (2005) also investigated the passenger loading process using simulation. Different boarding strategies were examined. Their particular contribution was the inclusion of disturbances, which could affect the speed of the loading process. These included situations where passengers did not follow announced boarding groups.

Combination linear programming and simulation model approaches have also been utilized for the aircraft loading process. The research by Van Del Briel, Villalobos, and Hogg (2003) and Van Del Briel, Villalobos, Hogg, Lindemann, and Mule (2005) focused on a fixed six-seat across layout for the Airbus 320 model aircraft, with three seats on either side of a single aisle. With this model aircraft, the researchers found that a dynamic hybrid outside-in and back- to-front, reverse-pyramid approach to passenger loading was most effective.
A physics based approach to the loading process has also been examined by Bachmat, Berend, Sapir, Skiena, and Stolyarov (2006). A significant difference between this research and other existing efforts is the focus on a theoretical approach rather than an empirical simulation based approach. A weakness of this theoretical approach is the absence of analysis from a seating configuration design standpoint. Regardless of the difference in approaches, the major contribution of this paper is the conclusion that under certain circumstances, many back-to-front loading policies are ineffective.

In 2009, Givoni and Rietveld investigated the phenomenon of the use of more frequent flights with limited capacity aircraft rather than the use of increased aircraft capacity to respond to increased travel demands. Their research confirmed the commercial air transportation industry's interest in the effectiveness and flexibility of the use of lower capacity aircraft with respect to level of demand and level of competition.

In summary, the significant research efforts to date focus on different boarding strategies including back to front, outside-in, and hybrid combination approaches. These strategies are based on six-seat-across configurations with a single passenger aisle. As these strategies are limited to this "standard" configuration, possible improvements in passenger loading as a result of fundamentally different seating configurations are ignored.

\section{Problem Statement}

As described in the previous section, the focus of previous research is on the best loading practices of existing common commercial seating configurations. In contrast, the objective of this research effort is to specifically explore and raise an awareness of the potential improvements in passenger loading as a result of different possible seating configurations in commonly utilized lower capacity commercial aircraft.

\section{Research Methodology}

The research methodology consisted of system definition, input data collection and analysis, model translation, verification and validation, experimental design, and output analysis.

\section{System Definition}

Commercial passenger aircraft are available in an exhaustive list of passenger configurations. Stretch versions, number of classes, and seat pitch result in an infinite number of configurations. To allow meaningful comparisons between passenger seating designs, a standard plane passenger capacity was selected. This was based on the same type of "standard" plane examined in previous simulation studies that consists of the six-seat-across configuration with a single 
aisle. with 20 rows, the capacity of this plane consisted of 120 seats. For the purposes of this study, the design configurations will be restricted to this same capacity. Four different seating configurations were analyzed; these included the standard configuration and three additional configurations based on the number of rows, the number of seats, and the number of aisles.

To ensure the validity of the experimental analysis, both the effects of the number of carry-on bags and seating related interferences were incorporated into the model. The number of carry-on bags can influence loading time due to aisle blockage caused by placing bags in the overhead bins. Similarly, the positioning of the passenger relative to previously seated passengers can also influence the loading time. If passengers must unseat themselves to allow other passengers to become seated, the aisle also becomes blocked.

To reduce the number of experimental variables only one commonly-utilized seating strategy was incorporated into the model. This strategy was random passenger seating starting with the back third, then the middle third, and finally the front third. No requirement was made that the passengers load outside to inside. Passengers were allowed between zero and two carry-on bags.

\section{Input Data Collection and Analysis}

Input data was collected for the number of passenger carry-on bags, the time required to secure various numbers of carry-on bags, the time required for passengers to become seated, and the duration of passenger aisle blockages due to previously seated passengers. A minimum of thirty data points were obtained for each type of input data. Data were also collected for the entire passenger loading time for validation purposes.

\section{Model Translation}

The system was translated using the simulation software Arena. This is a graphical simulation software package distributed by Rockwell Software. The translation consists of developing a model, an experiment, and an animation. The model incorporates a series of modules, and is similar to a detailed flowchart. The experiment consists of parameter values for the model components. The animation provides a graphical representation of the model.

\section{Model Validation}

Model validation consisted of statistically comparing the loading times between an actual aircraft and the simulation model. The collection of actual aircraft system data was complicated by post $9 / 11$ security measures. As a result for the complete passenger loading process only eight aircraft were able to be observed for validation purposes. Due to the less than optimal number of complete loading process observations, a non-parametric rank sum test was required for the validation process.

The mean and standard deviation of the loading time for both the system and the base model are listed in Table 1.

The following section summarizes the non-parametric validation process.

- $\mathrm{H}_{\mathrm{o}}$ : The mean loading time between the system and the model is not statistically different at the given alpha level

- $\mathrm{H}_{\mathrm{a}}$ : The mean loading time between the system and the model is statistically significantly different at the given alpha level.

- Level of Significance, alpha $=0.05$

- Critical Value for alpha $=0.05$ is \pm 1.96 using the $\mathrm{Z}$ distribution

- Test Statistic. The Rank Sum Test yielded a test statistic of -0.95

- Decision. The test statistic of -0.95 was between -1.96 and 1.96. The $\mathrm{H}_{\mathrm{o}}$ cannot be rejected.

The fact that the $\mathrm{H}_{\mathrm{o}}$ cannot be rejected at an alpha level of 0.05 provides support for the claim that the base model is statistically valid. With a statistically valid base model, the experimental alternatives were developed.

\section{Experimental Design}

As described in the system definition section, a total of four configurations were examined based on the number of rows, seats across, and number of aisles. Each configuration was assigned a code that represented its particular characteristics. The first two digits of configuration codes are the number of rows in the configuration. The third digit is the number of seats across. The last digit corresponds to the number of aisles. The four configurations were:

- Configuration 2061: Base model with 20 rows; six seats, with three on either side; and one aisle.

- Configuration 2451: New design configuration with 24 rows of five seats, with two seats on one side and three seats on the either side of the single aisle.

- Configuration 3021: New design configuration with 30 rows of four seats, with two seats on either side of a single aisle.

- Configuration 2062: New design configuration with 20 rows of six seats, with two seats on either side and two seats in the center between two aisles.

Table 1

Model Validation Data

\begin{tabular}{lcc}
\hline Data Source & Mean Time in Minutes & Standard Deviation in Minutes \\
\hline System & 19.08 & 2.98 \\
Model & 20.34 & 1.38 \\
\hline
\end{tabular}


Table 2

Load Time Summary Statistics and Replication Requirements

\begin{tabular}{lcccc}
\hline Configuration & Mean Load Time in Seconds & Standard Deviation of Load Time in Seconds & Replications Required & Relative Precision \\
\hline 2061 & 1220 & 49.9 & 10 & 0.029 \\
2451 & 1200 & 69.0 & 10 & 0.041 \\
3041 & 1110 & 52.1 & 10 & 0.034 \\
2062 & 672 & 47.6 & 10 & 0.051 \\
\hline
\end{tabular}

\section{Replication Analysis}

Since the passenger loading time is probabilistic, replication analysis is required in order to make a statistically robust comparison between configurations. This consists of determining the number of simulation runs or replications that is necessary at a given level of confidence. The relative precision method of replication analysis was utilized for this study. This method consists of calculating the ratio of the half-width confidence interval over the mean of the replication data.

Table 2 indicates that 10 replications were sufficient for each configuration to achieve a desired relative precision ratio of 0.10 .

\section{Discussion}

The output data from the four alternatives were examined using Analysis of Variance. This consisted of the following steps.

- $\mathrm{H}_{\mathrm{o}}$ : There is no statistically significant difference between the means at the given alpha level.

- $\mathrm{H}_{\mathrm{a}}$ : There is a statistically significant difference between the means at the given alpha level.

- Level of significance, alpha $=0.05$

- Critical Value. The critical value for the F distribution for a numerator with three degrees of freedom and a denominator of 36 degrees of freedom, at an alpha level of 0.05 is 2.866 .

- Test Statistic. The test statistic for the ANOVA test was 218.081. The ANOVA results are summarized in Table 3.

- Decision. Since the test statistic is greater than the critical value, the Ho must be rejected. This means that there is evidence to support the fact that there is a difference in loading performance between at least one of the configurations.

Table 3

ANOVA Results

\begin{tabular}{lcccc}
\hline $\begin{array}{l}\text { Source of } \\
\text { Variation }\end{array}$ & $\begin{array}{c}\text { Sum of } \\
\text { Squares }\end{array}$ & $\begin{array}{c}\text { Degrees of } \\
\text { Freedom }\end{array}$ & $\begin{array}{c}\text { Mean } \\
\text { Squares }\end{array}$ & F Value \\
\hline Between & $2.001 \mathrm{e}+006$ & 3 & $6.669 \mathrm{e}+005$ & 218.081 \\
Error (Within) & $1.101 \mathrm{e}+005$ & 36 & $3.06 \mathrm{e}+003$ & \\
Total & $2.111 \mathrm{e}+006$ & 39 & & \\
\hline
\end{tabular}

To obtain additional insight into these results, a Duncan Multiple Range test was performed on the alternatives. This test calculates a least significant range value $\mathrm{R}$ for each set of adjacent means. If the range of the adjacent means exceed the critical value, the adjacent means are statistically significantly different. Table 4 lists the least significant range values.

Table 5 summarizes the results of the Duncan Multiple Range Test. The underline between adjacent means signifies a non-significant range.

The results presented in Table 5 signify that there is no significant difference between configurations 2061 and 2451 at an alpha level of 0.05 . However, configuration 2061 is statistically significant from both configurations 3041 and 2062. Configuration 2451 is also statistically significantly different from configurations 3041 and 2062. Lastly, configuration 3041 is statistically significantly different from configuration 2062.

\section{Conclusions}

Since there was no statistically significant difference between the base configuration 2061 of 20 rows, six seats across, with a single aisle and configuration 2451 of 24 rows, five seats across, with a single aisle, no consideration should be given to further investigation of configuration 2451. The 2451 configuration may also be disturbing to both aircraft designers and passengers alike due to its asymmetric design.

However, both configurations 3041 and 2062 are statistically significantly different from the base configuration 2061. The difference between configuration 3041 and configuration 2061 means that reducing the number of

Table 4

Duncan Multiple Range Values At Alpha $=0.05$

\begin{tabular}{lc}
\hline Number of Adjacent Means & Least Significant Range in Seconds \\
\hline 2 & 50.55 \\
3 & 53.00 \\
4 & 54.75 \\
\hline
\end{tabular}

Table 5

Duncan Multiple Range Test Results

\begin{tabular}{llllr}
\hline Configuration & 2061 & 2451 & 3041 & 2062 \\
Load Time & 1220 & 1200 & 1110 & 672 \\
\hline
\end{tabular}


seats on either side of the aisle can make a difference in loading times. This difference is a result of the reduced number of previously seated passenger related aisle interferences. Configuration 2062 shows particular promise, as both previously seated passenger related aisle interferences, as well as luggage placement interferences, are reduced by virtue of having two aisles and only two seats on either side of the aisles.

The results obtained from this research effort should not be taken to the exclusion of other aircraft design parameters. For example, the 3041 four-seat-across configuration allows for an approximate nine percent reduction in the loading time over the base configuration. However, it will require a longer fuselage than other configurations. This will allow either the width across the fuselage to be reduced or permit the use of wider seats. If a narrower fuselage is selected, the cross-section will be correspondingly smaller.

Similarly, the use of a necessarily wider fuselage for the 2062 two-aisle configuration allows an approximate 45 percent reduction in the passenger loading process time. However, this design will necessarily result in a larger cross sectional area than other configurations. This in turn could lead to issues related to less efficient aerodynamics.

In summary, statistically significant advantages in passenger loading time may be achieved by reducing the number of seats on either side of the aisle from three to two. Statistically significant loading advantages may also be obtained by increasing the number of aisles in the fuselage. However, as is the case with many other design parameters, increased loading benefits will have to be weighed against possible design disadvantages.

\section{References}

Bachmat, E., Berend, D., Sapir, L., Skiena, S., \& Stolyarov, N. (2006). Analysis of aeroplane boarding via spacetime geometry and random matrix theory. Journal of Physics: Mathematical and General, 39, L453-L459.

Givoni, M., \& Rietveld, P. (2009). Airline's choice of aircraft size explanations and implications. Transportation Research Part A: Policy and Practice, 43(5), 500-510.

Ferrari, P., \& Nagel, K. (2005). Robustness of efficient passenger boarding in airplanes. Transportation Research Record, 1915, 44-54.

Marelli, S., Mattocks, G., \& Merry R. (1998). The role of computer simulation in reducing airplane turn time. AERO Magazine, 1(1). Retrieved from www.boeing.com/commercial/aeromagazine/aero_01/ textonly/t01txt.html

McLean, D. (2006). The operational efficiency of passenger aircraft. Aircraft Engineering and Aerospace Technology, 78(1), 32-38.

Van Del Briel, M., Villalobos, J., Hogg, G., Lindemann T., \& Mule, A. (2005). America West Airlines develops efficient boarding strategies. Interfaces, 35(3), 191-203.

Van Del Briel, M., Villalobos, J., \& Hogg, G. (2003). The aircraft loading problem. Proceedings of the $12^{\text {th }}$ Industrial Engineering Research Conference, paper 2153, Portland, Oregon.

Van Landeghem, H., \& Beuselinck, A. (2002). Reducing passenger boarding time in airplanes: a simulation based approach. European Journal of Operations Research, 142, 294-308. 